\title{
Dependencia emocional, satisfacción con la vida y violencia de pareja en estudiantes universitarias
}

\section{Emotional Dependence, Satisfaction with Life and Partner Violence in University Students}

\author{
Carlos Ramón Ponce-Díaz* \\ Universidad Inca Garcilaso de la Vega, Lima, Perú \\ Universidad San Ignacio de Loyola, Lima, Perú \\ ORCID: http://orcid.org/0000-0002-0101-2244 \\ Jesús Joel Aiquipa Tello \\ Universidad Inca Garcilaso de la Vega, Lima, Perú \\ ORCID: http://orcid.org/0000-0002-1982-6908 \\ Manuel Arboccó de los Heros \\ Universidad Inca Garcilaso de la Vega, Lima, Perú \\ ORCID: http://orcid.org/0000-0002-8481-310X
}

Recibido 03-06-19 Revisado 05-08-19 Aprobado 13-11-19 En línea 26-11-19

*Correspondencia

Email: cponcediaz@hotmail.com
Citar como:

Ponce-Díaz, C., Aiquipa, J., \& Arboccó, M. (2019). Dependencia emocional, satisfacción con la vida y violencia de pareja en estudiantes universitarias. Propósitos y Representaciones, 7(SPE), e351. http://dx.doi.org/10.20511/pyr2019.v7nSPE.351 


\section{Resumen}

El objetivo del estudio fue analizar la relación entre la dependencia emocional y la satisfacción con la vida en un grupo de estudiantes de Psicología de universidades públicas y privadas de Lima Metropolitana, víctimas y no-víctimas de violencia de pareja. Participaron 1211 estudiantes universitarias con edad promedio de 21.7 años $(\mathrm{DE}=4,7), 54 \%$ sin violencia de pareja y $46 \%$ con violencia. Los instrumentos empleados fueron el Cuestionario de Violencia entre Novios (CUVINO, Rodríguez-Franco et al., 2010), el Inventario de Dependencia Emocional (IDE, Aiquipa, 2015) y la Escala de Satisfacción con la Vida (SWLS, Diener, Emmons, Larsen \& Griffin, 1985). Los resultados indican que en el grupo de mujeres con violencia de pareja existe relación estadísticamente significativa y de tipo inversa entre dependencia emocional y satisfacción con la vida, tanto para la escala total $(-0,24 ; p<.005)$ como para todas las dimensiones (valores entre $-0,128$ hasta $-0,26 ; p<.001$ ). Sin embargo, en el grupo de mujeres sin violencia de pareja, la relación entre las variables de estudio fue menor $(-0,11 ; \mathrm{p}<.005)$ y solo se halló relación entre satisfacción con la vida y tres de las siete dimensiones (valores entre $-0,11$ hasta $-0,16 ; \mathrm{p}<.005)$.

Palabras clave: Violencia de pareja; Dependencia emocional; Satisfacción con la vida; Estudiantes universitarias.

\section{Summary}

The objective of the study was to analyze the relationship between emotional dependence and satisfaction with life in a group of Psychology students from public and private universities in Metropolitan Lima, victims and non-victims of partner violence. The research involved 1211 university students with an average age of 21.7 years $(\mathrm{SD}=4.7), 54 \%$ not subjected to any partner violence and $46 \%$ who had experienced violence. The instruments used were the Questionnaire on Violence between partners (CUVINO, Rodríguez-Franco et al., 2010), the Inventory of Emotional Dependence (IDE, Aiquipa, 2015) and the Satisfaction with Life Scale (SWLS, Diener, Emmons, Larsen \& Griffin, 1985). The results indicate that in the group of women experiencing partner violence there is a statistically significant relationship and an inverse relationship between emotional dependence and life satisfaction, both for the total scale $(-0.24, \mathrm{p}<.005)$ and for all dimensions (values between -0.128 to $-0.26, \mathrm{p}<.001$ ). However, in the group of women not subjected to partner violence, the relationship between the study variables was lower $(-0.11, p$ $<.005)$ and some relationship was only found between satisfaction with life and three of the seven dimensions (values between -0.11 to $-0.16, \mathrm{p}<.005$ ).

Keywords: Partner Violence; Emotional Dependence; Satisfaction with Life; University Students.

\section{Introducción}

La violencia de pareja se define como cualquier comportamiento dentro de una relación íntima que causa daño físico, psicológico o sexual a los miembros de la relación. Dicho comportamiento puede incluir agresión física, abuso psicológico, relaciones sexuales forzadas y comportamientos de control (Heise \& García-Moreno, 2003). Asimismo, la mayoría de las víctimas de este tipo de violencia en las relaciones heterosexuales son mujeres, siendo los hombres quienes la perpetran (United Nations, 2015).

En los últimos años, el interés por estudiar la violencia que se ejerce contra la mujer, en jóvenes y adolescentes, se ha incrementado (Cortés-Ayala et al., 2015; Rodríguez-Franco et al., 2016; Stöckl, March, Pallitto, \& Garcia-Moreno, 2014; Viejo, 2014). No es que se suspendió indagar esta problemática en mujeres adultas que ya cohabitan con la pareja, sino que, al parecer, esta tendencia pretende indagar oportunamente las características de este tipo de violencia y las 
variables asociadas que pudieran explicarla, de tal forma que se optimicen las intervenciones y reduzcan los efectos perjudiciales en las mujeres. Asimismo, es importante señalar que la mayoría de los estudios de violencia de pareja en el noviazgo se realizaron con estudiantes universitarias (Aizpurua, Caravaca-Sánchez \& Stephenson, 2018; García et al., 2013; López-Cepero, Lana, Rodríguez-Franco, Paíno \& Rodríguez-Díaz, 2015).

En el contexto peruano, el Instituto Nacional de Estadística e Informática (INEI) a través de la Encuesta Demográfica y de Salud Familiar (ENDES), reporta que a nivel nacional el $65.4 \%$ de las mujeres entre 15 a 49 años, alguna vez, sufrieron algún tipo de violencia por parte del esposo o compañero (INEI, 2017). De forma más precisa, Vara-Horna et al. (2016) encontraron que el $65 \%$ de estudiantes mujeres universitarias que tuvieron pareja, alguna vez en sus vidas, fueron atacadas por sus parejas o exparejas, mientras que, en el último año precedente al estudio, el $47.8 \%$ de mujeres fue agredida por su pareja, con un promedio de 20 ataques por año.

Las consecuencias perjudiciales reportadas en estudiantes universitarias que son víctimas de violencia de pareja implican, entre otros, problemas de salud mental como estrés, trastornos ansiosos, depresión e ideas suicidas (Aizpuru et al., 2018; Eshelman \& Levendosky, 2012; Martín-Baena, Talavera, \& Montero-Piñar, 2016) y dificultades en el rendimiento académico (Banyard et al., 2017). Respecto a las variables asociadas con el inicio y mantenimiento de la violencia de pareja en jóvenes, es importante mencionar que la mayoría de los estudios coindicen en agruparlas en factores de riesgo demográficos, contextuales, familiares, relacionales y psicológicos (Capaldi, Knoble, Shortt, \& Kim, 2012). Así, se reporta, entre otras variables, experiencias previas de violencia familiar (Stöckl et al., 2014); dificultades en la regulación emocional (Momeñe, Jáuregui, \& Estévez, 2017); autoestima disminuida (Moral, García, Cuetos, \& Sirvent, 2017) y autoeficacia en la relación (Baker, Cobb, Mcnulty, Lambert, \& Fincham, 2016).

Los estudios revisados tanto en población adulta como en jóvenes reportan relación entre dependencia emocional y violencia en el noviazgo (Del Castillo, Hernández, Romero, \& Iglesias, 2015, Momeñe et al., 2017; Moral et al., 2017). Otros estudios encuentran niveles altos de dependencia emocional en aquellas mujeres que reportaron maltratos por parte de sus parejas respecto a sus pares que no sufrían dicha violencia, resaltando que las características como priorización de la pareja, sumisión y subordinación y miedo a la ruptura, parecen ser atributos principales de la dependencia emocional en mujeres que reciben malos tratos (Aiquipa, 2015; Huerta et al., 2016).

En este contexto, una de las variables asociadas a la violencia de pareja es la dependencia emocional. En los últimos años, este constructo es objeto de interés e investigación en el ámbito de las relaciones de pareja (Avci Çayir \& Kalkan, 2018; Estévez et al., 2018; Gonzalez-Bueso et al., 2018; Niño \& Abaunza, 2015; Urbiola \& Estévez, 2015; Urbiola, Estévez, Iruarrizaga, \& Jauregui, 2017). Si bien la literatura presenta diversas teorías que tratan la dependencia emocional, para efectos del presente estudio se utiliza la propuesta de Castelló (2005). El autor propone el modelo de vinculación afectiva para explicar la naturaleza de la dependencia emocional. Así, refiere que los seres humanos tienen la necesidad de vincularse con los otros a fin de obtener seguridad y afecto, lo cual genera lazos permanentes con ellas. Esta vinculación afectiva con otros puede darse en diferentes grados. Para el autor, la diferencia entre las relaciones de parejas normales y sus pares dependientes emocionales, son continuas, pudiendo ubicarse en un hipotético continuo en grados o niveles de vinculación afectiva. La dependencia emocional constituye la dimensión extrema de la necesidad de vinculación afectiva, generándose un patrón de pensar, sentir y actuar más o menos estable en la persona en la interacción con sus parejas.

Por otro lado, si bien hay estudios que indagaron la relación entre bienestar psicológico y violencia de pareja (Bernardo \& Estrellado, 2017; Mohamed \& Herrera, 2014; Montero et al., 2011; Poutiainen \& Holma, 2013), solo algunos estudios abordan específicamente la satisfacción con la vida en mujeres que sufren violencia de pareja, y los que hay proponen que una de las 
consecuencias de este tipo de violencia es justamente la disminución del nivel de satisfacción con la vida (Callahan, Tolman, \& Saunders, 2003; García, Wlodarczyk, Reyes, San Cristóbal, \& Solar, 2014).

En este contexto, la satisfacción con la vida constituye uno de los componentes del bienestar subjetivo y se define como la evaluación cognitiva y global de la calidad de la vida en general (Diener, Emmons, Larsen, \& Griffin, 1985). De esta forma, la satisfacción con la vida representa un juicio evaluativo (Pavot \& Diener, 2008). Según Schimmack, Diener y Oishi (2002), los cambios en la satisfacción con la vida suelen estar sistemáticamente ligados a los cambios en dominios específicos y accesibles, en lugar de ser el producto de factores contextuales aleatorios. En consecuencia, los juicios de satisfacción con la vida parecen ser sensibles a los cambios en los dominios importantes de la vida de una persona. Así, algunos estudios identifican dominios importantes que pueden influir en los juicios de satisfacción con la vida, tales como apoyo social (Diener \& Diener McGavran, 2008; Turner \& Brown, 2010; Novoa \& Barra, 2015; Ruvalcaba-Romero, Fernández-Berrocal, Salazar-Estrada, \& Gallegos-Guajardo, 2017; Trepte, Dienlin, \& Reinecke, 2015), desempeño académico (Pavot \& Diener, 2008; Schimmack et al., 2002), objetivos o metas personales (Heckhausen \& Kay, 2018; Klug \& Maier, 2015) y actividades de entretenimiento y de ocio (Liu \& Yu, 2015; Pascucci, 2015).

No se ha encontrado investigación que reporte la posible relación entre dependencia emocional y satisfacción con la vida en el contexto de violencia de pareja. En ese sentido, los autores consideran que la temática planteada es de actualidad y de interés nacional y además su estudio se justifica ampliamente por los numerosos casos de violencia de pareja y feminicidios que han ocurrido en los últimos años en el Perú, donde un grupo de esos casos fueron precisamente estudiantes universitarias.

El papel formativo de las universidades es de suma importancia para el desarrollo profesional de los estudiantes. De allí que en muchas instituciones de educación superior se desarrollan planes tutoriales orientados al desarrollo de aspectos personales (autoestima, habilidades sociales, regulación de emociones, entre otros), con el objetivo de fomentar una mejor adaptación de los educandos. En este sentido, el presente estudio se enmarca en el campo de estudio de las variables no cognitivas asociadas al rendimiento académico toda vez que su presencia puede influir de manera desfavorable o negativa en la consolidación personal y profesional del estudiante.

Por cuanto se ha mencionado, el objetivo principal del estudio es analizar la relación entre la dependencia emocional y la satisfacción con la vida en estudiantes universitarias de psicología de Lima Metropolitana, víctimas y no-víctimas de violencia de pareja. Para lograr ello, en primer lugar, se identifica el porcentaje de mujeres que reportaron ser víctimas de violencia de pareja; luego, se comparan los puntajes de dependencia emocional y satisfacción con la vida en los dos grupos (con violencia y sin violencia) y finalmente se analiza el tipo e intensidad de relación entre las variables estudiadas.

\section{Método}

El estudio es una investigación empírica con metodología cuantitativa. Siguiendo a Ato, López y Benavente (2013) este estudio se corresponde con la estrategia asociativa, en la medida que se exploró la relación entre las variables satisfacción con la vida y dependencia emocional en los grupos con y sin violencia de pareja; y transversal, pues el estudio se definió en un momento temporal determinado.

\section{Participantes}

Participaron 1211 mujeres mayores de 18 años, estudiantes universitarias de la Carrera de Psicología, residentes en Lima, que tuvieron por lo menos una relación de pareja heterosexual. El 
43\% de las participantes provienen de universidades públicas, mientras que el $67 \%$ son de universidades privadas. La edad promedio del grupo es de 21.7 años $(D E=4.7)$ y su estado civil predominante es soltera (95\%). El 74\% no trabaja mientras que 26\% comparte sus estudios con actividad laboral. La mayoría de ellas cohabitan con sus padres (78\%), 8.7\% vive con otros familiares, $9.2 \%$ vive sola y solo un $3.8 \%$ cohabita con su pareja. El $94 \%$ no tiene hijos y el $46 \%$ tiene pareja en la actualidad mientras que el $54 \%$ no lo tiene.

\section{Instrumentos}

Ficha de datos sociodemográficos. Se elaboró un cuestionario ad hoc que contenía preguntas para recabar datos como edad, lugar de residencia, tipo de universidad, estado civil, cohabitación, situación laboral y relación de pareja heterosexual actual.

Cuestionario de Violencia entre Novios-CUVINO (Rodríguez-Franco et al., 2010). La prueba consta de 42 reactivos en formato Likert de cinco opciones (entre 0 a 4) que evalúa comportamientos que indican violencia en las relaciones sentimentales de pareja en edad adolescente y joven. Cada uno de los reactivos indaga sobre la presencia de esos indicadores y sobre el grado de molestia que ocasionó en la persona esos comportamientos. De acuerdo con el propósito del estudio, solo se consideró la presencia de los indicadores a fin de identificar a aquellas estudiantes que reportaron violencia de pareja y quienes no lo hicieron. Las propiedades psicométricas reportadas indican un coeficiente de confiabilidad alfa de Cronbach igual a 0.93 para la escala total y valores entre 0.58 y 0.81 para sus ocho dimensiones; ítems con cargas factoriales entre 0.36 y 0.76 , compuesto por 8 factores que explican el $51.3 \%$ de la varianza total y correlaciones estadísticamente significativas entre todos los factores, con intensidad de relación de 0.30 a 0.65 . Para la presente investigación, se realizó un estudio piloto con 300 mujeres estudiantes universitarias (características similares a la muestra final), a fin de corroborar la pertinencia y rigurosidad de las pruebas psicológicas. De esta forma, el CUVINO obtuvo un coeficiente de confiablidad $\alpha=0.96$ para la escala total y valores entre 0.52 y 0.92 para sus factores; correlación ítem-test entre 0.33 a 0.79 y varianza explicada por los ocho factores de $69.7 \%$.

Inventario de Dependencia Emocional-IDE (Aiquipa, 2012, 2015). Este instrumento está constituido por 49 ítems en escala tipo Likert de cinco alternativas (entre 1 y 5) y evalúan comportamientos de dependencia afectiva hacia la pareja). El instrumento obtuvo indicadores de confiabilidad satisfactorios como $\left(\alpha=0.96 ; r_{c}=0.91\right)$. Asimismo, se halló evidencias de validez de constructo adecuados, tales como índices de acuerdo con valores de .80 y 1.00 para más del 95\% de los reactivos, reproducción de 7 factores que explicaron el $58.3 \%$ de la varianza total, conteniendo ítems con cargas factoriales entre 0.40 y 0.70 y diferencias de medias entre grupo clínico y no clínico significativa al 95\% de nivel de confianza. En el estudio piloto $(\mathrm{n}=300)$, el IDE obtuvo un coeficiente de confiablidad $\alpha=0.97$ para la escala total y valores entre 0.79 y 0.90 para sus factores; correlación ítem-test entre 0.32 a 0.74 y varianza explicada por los siete factores de $61.5 \%$.

Escala de Satisfacción con la Vida-SWLB (Diener et al., 1985). Compuesta por cinco ítems redactados en dirección positiva mediante escala de respuesta Likert de 7 puntos (desde 1 = "muy en desacuerdo" hasta a 7 = "totalmente de acuerdo"). Las propiedades psicométricas iniciales del SWLB reportaron un coeficiente de correlación test-retest (dos meses) de 0.82 y coeficiente alfa 0.87 ; reproducción de un solo factor que representa el $66 \%$ de la varianza, con ítems con cargas factoriales entre 0.61 y 0.84 . Diversos estudios han confirmado las propiedades psicométricas satisfactorias del SWLB, en diferentes muestras de estudio, tanto internacionales (Atienza, Pons, Balaguer, \& García-Merita, 2000; Cecilia, Martínez, \& Pilar, 2013; Celis-Atenas, \& Silva, 2012; López-Ortega, Torres-Castro, \& Rosas-Carrasco, 2016; Padrós, Gutiérrez, \& Medina, 2015; Pavot \& Diener, 2008; Vera-Villarroel, Urzúa, Pavez), como nacionales (Alarcón, 2000; Arias-Gallegos, Huamani-Cahua, \& Caycho-Rodríguez, 2018; Caycho-Rodríguez et al., 2018). Los resultados del estudio piloto $(n=300)$ indican que el SWLB obtuvo un coeficiente de 
confiablidad $\alpha=0.81$; correlación ítem-test entre 0.58 a 0.72 y modelo unidimensional que explicó el $58.7 \%$ de la varianza de los puntajes.

\section{Procedimiento}

Una vez seleccionados los instrumentos de medida, se solicitó de forma virtual la autorización para el uso de éstos a los autores respectivos, quienes respondieron favorablemente. Se elaboró el consentimiento informado y se editaron los instrumentos de tal modo que formen un solo cuadernillo. Paralelamente, los miembros del equipo se contactaron con colaboradores clave para la aplicación de los instrumentos. Estos colaboradores fueron psicólogos y catedráticos de universidades públicas y privadas, quienes tenían a su cargo varios grupos de estudiantes. En ese sentido, la recolección de información se realizó de forma presencial en las aulas de clase de las participantes de las diferentes universidades, entre mayo y julio de 2018, siguiendo las instrucciones estandarizadas de las pruebas y confirmando la participación voluntaria de las estudiantes a través de la firma del consentimiento informado. Tras una revisión exhaustiva de los formatos aplicados, se eliminaron 75 de ellos pues estaban incompletos. Luego, se elaboró la base de datos con el programa Microsoft Excel para su posterior análisis estadístico.

\section{Análisis de datos}

Se utilizaron estadísticas descriptivas, tales como medidas de dispersión (desviación estándar), tendencia central (media y mediana) y distribución (índices de asimetría y curtosis) para caracterizar las variables de estudio. Para examinar la diferencia de grupos se usó el estadístico no paramétrico $U$ de Mann- Whitney y la probabilidad de superioridad o PS como indicador del tamaño de efecto (Grissom, 1994; Grissom \& Kim, 2012; Newcombe, 2006), pudiendo ser estos valores pequeño ( $P S \geq 0.56$ ), mediano $(P S \geq 0.64)$ y grande $(P S \geq 0.71)$ (Grissom, 1994). Asimismo, se realizó análisis bivariado a través del coeficiente rho de Spearman $(\rho)$, tomándose el valor de la intensidad del coeficiente como indicador del tamaño del efecto de dicha relación (Cohen, 1988; Ferguson, 2009). De esta forma, se consideró valores $\rho \geq 0.10$ como pequeño, $\rho \geq$ 0.30 mediano y $\rho \geq$ a 0.50 como grande (Cohen, 1988; Grissom, 1994). Para todos los análisis se utilizó un nivel de significancia de 0.05 . El procesamiento de datos se realizó con el apoyo del programa IBM SPSS Statistics 24.0.

\section{Resultados}

En la Tabla 1 se muestra los estadísticos descriptivos de las variables de estudio. Se observa que la media de la muestra en satisfacción con la vida fue $25.5(D E=5)$, mientras que el grupo total de estudiantes obtuvo en la escala total de dependencia emocional un promedio de 82 puntos ( $D E$ $=28.7$ ).

El puntaje promedio en la variable violencia de pareja fue $10.8(D E=17.3)$. Para determinar los grupos de mujeres con y sin indicadores de violencia de pareja, se estableció como punto de corte el puntaje correspondiente al percentil 55 (6 puntos), pues el análisis ad hoc de diferencia de rangos de puntajes realizadas en el estudio, indicó que los grupos así formados presentaron diferencias estadísticamente significativas en el puntaje total de violencia de pareja $(U=364650, p<0.01)$. Asimismo, es importante considerar la recomendación de los autores del CUVINO respecto a que cualquiera de las conductas contempladas en el mismo, incluso solo una, pueden ser indicadores de violencia (Rodríguez-Franco et al., 2010). Así, el grupo sin violencia estuvo constituido por mujeres que tuvieron de 0 a 5 puntos, lo que hace un total de 650 casos (54\%), mientras que el grupo con violencia tuvo de 6 a más puntos, contabilizándose 561 casos $(46 \%)$.

Por otro lado, se encontró que los puntajes de las variables dependencia emocional y violencia de pareja presentaron índices de asimetría y curtosis fuera del rango \pm 1 (George \& 
Mallery, 2016), concluyendo que dichos puntajes no se aproximan a una distribución normal, por lo que es pertinente el empleo de pruebas no paramétricas para los siguientes análisis.

Tabla 1.

Estadísticos descriptivos de violencia de pareja, satisfacción con la vida y dependencia emocional

\begin{tabular}{lccccc}
\hline \multirow{2}{*}{\multicolumn{1}{c}{ Variables }} & \multicolumn{3}{c}{ Descriptivos } & \multicolumn{2}{c}{ Normalidad } \\
\cline { 2 - 6 } \multicolumn{1}{c}{$M$} & $M d n$ & $D E$ & $\mathrm{~g} 1$ & $\mathrm{~g} 2$ \\
\hline Violencia de pareja & 10.8 & 5 & 17.3 & 3.1 & 11.9 \\
Satisfacción con la vida & 25.5 & 26 & 5.0 & -0.9 & 1.0 \\
Dependencia emocional & 82.0 & 75 & 28.7 & 1.7 & 3.6 \\
Miedo a la ruptura & 12.5 & 10 & 5.3 & 2.5 & 7.7 \\
Miedo e intolerancia a la soledad & 18.3 & 16 & 7.1 & 1.5 & 2.7 \\
Prioridad de pareja & 12.3 & 10 & 5.4 & 2.0 & 4.4 \\
Necesidad de acceso a la pareja & 12.8 & 12 & 4.6 & 0.7 & 0.4 \\
Deseos de exclusividad & 7.8 & 7 & 3.3 & 1.8 & 4.1 \\
Subordinación y sumisión & 9.9 & 9 & 3.9 & 0.9 & 0.6 \\
Deseos de control y dominio & 8.3 & 7 & 3.6 & 1.6 & 3.0 \\
\hline
\end{tabular}

Nota: $\mathrm{N}=1211$. M: media; Mdn: mediana; DE: desviación estándar; g1: Asimetría; g2: Curtosis

La Tabla 2 muestra el rango promedio de puntajes, estadístico $U$ de Mann- Whitney y la Probabilidad de Superioridad (PS), para dependencia emocional (escala total y dimensiones) y satisfacción con la vida en los grupos con violencia de pareja y sin ella. Se observa que, en el grupo de mujeres con violencia de pareja, el rango promedio de puntajes en satisfacción con la vida es menor que en el grupo sin violencia. Respecto a la variable dependencia emocional (puntaje total y dimensiones), el grupo de mujeres con violencia de pareja presenta rangos promedios mayores que el grupo de mujeres sin violencia de pareja. Asimismo, a juzgar por el valor de la prueba no paramétrica $U$ de Mann- Whitney y los niveles de significancia correspondientes, existen diferencias estadísticamente significativas entre el grupo con violencia y sin violencia en los puntajes de satisfacción con la vida $(U=138509.5, p<0.01)$ y dependencia emocional, tanto en la escala total $(U=93023.5, p<0.01)$ como en las siete dimensiones $(U=$ 233321-277781.5, $p<0.01)$. La magnitud de esta diferencia es pequeña para satisfacción con la vida $(P S=0.38)$ y grande para la escala total de dependencia emocional $(P S=0.74)$ y entre mediano y grande para sus dimensiones $(P S=0.63-0.76)$. 


\section{Tabla 2.}

Diferencia en satisfacción con la vida y dependencia emocional en mujeres con o sin violencia de pareja

\begin{tabular}{|c|c|c|}
\hline & $\begin{array}{l}\text { Con violencia } \\
\quad(n=561)\end{array}$ & $\begin{array}{l}\text { Sin violencia } \\
\quad(n=650)\end{array}$ \\
\hline
\end{tabular}

Rango promedio Rango promedio

\begin{tabular}{lcccc}
\hline Satisfacción con la vida & 527.90 & 673.41 & $138509.5^{*}$ & 0.38 \\
Dependencia emocional (Total) & 765.18 & 468.61 & $271626.5^{*}$ & 0.74 \\
Miedo a la ruptura & 771.71 & 462.98 & $275286.5^{*}$ & 0.75 \\
Miedo e intolerancia a la soledad & 743.20 & 487.58 & $259297^{*}$ & 0.71 \\
Prioridad de pareja & 776.15 & 459.14 & $277781.5^{*}$ & 0.76 \\
Necesidad de acceso a la pareja & 696.90 & 527.54 & $233321^{*}$ & 0.63 \\
Deseos de exclusividad & 712.73 & 513.89 & $242198^{*}$ & 0.66 \\
Subordinación y sumisión & 739.06 & 491.16 & $256972.5^{*}$ & 0.70 \\
Deseos de control y dominio & 759.43 & 473.58 & $268396.5^{*}$ & 0.74 \\
\hline
\end{tabular}

Nota: $* p<0.01$. U: U de Mann- Whitney, PS: Probabilidad de Superioridad.

En la tabla 3 se observa que, en el grupo de mujeres con violencia de pareja, la relación entre dependencia emocional y satisfacción con la vida es estadísticamente significativa e inversa, tanto para el puntaje total como para cada una de las dimensiones de la variable dependencia emocional, con una intensidad de relación pequeña para la escala total $(\rho=-0.24)$ y sus dimensiones ( $\rho$ entre -.146 a -.256). Por otra parte, en el grupo de mujeres sin violencia, la variable satisfacción con la vida se asoció de forma inversa y estadísticamente significativa solo con cinco de los siete componentes de dependencia emocional, siendo la intensidad de dichas relaciones débil o pequeña para la escala total de dependencia emocional $(\rho=-0.10)$ y sus dimensiones (valores p entre -.087 y -.159).

Tabla 3.

Coeficiente de correlación rho de Spearman entre satisfacción con la vida y dependencia emocional en mujeres con y sin violencia de pareja

\begin{tabular}{lcc}
\hline \multirow{2}{*}{ Dependencia emocional } & \multicolumn{2}{c}{ Satisfacción con la vida } \\
\cline { 2 - 3 } & Con violencia $(n=561)$ & Sin violencia $(n=650)$ \\
\hline Puntaje total & $-.241^{* *}$ & $-.106^{* *}$ \\
Miedo a la ruptura & $-.252^{* *}$ & $-.159^{* *}$ \\
Miedo e intolerancia a la soledad & $-.256^{* *}$ & $-.161^{* *}$ \\
Prioridad de pareja & $-.229^{* *}$ & $-.111^{* *}$ \\
Necesidad de acceso a la pareja & $-.128^{* *}$ & -.033 \\
Deseos de exclusividad & $-.146^{* *}$ & -.046 \\
Subordinación y sumisión & $-.157^{* *}$ & -.054 \\
Deseos de control y dominio & $-.229^{* *}$ & $-.087^{* *}$ \\
\hline
\end{tabular}

Nota: $* * p<0.01 ; * p<0.05$ 


\section{Discusión}

Los resultados muestran que un $46 \%$ de las participantes reportan indicadores de violencia por parte de la pareja. Estos indicadores abarcan, por ejemplo, indiferencia, hacia los sentimientos de las participantes, críticas personales, conductas de burla por la condición de ser mujer, conductas manipuladoras, culpabilizar a la pareja por el estado de la relación, comportamientos para generar sufrimiento a la pareja como reclamos o muestras de enfado, amenazas de terminar la relación, agresiones físicas como empujones o bofetadas, e incluso actos y tocamientos sexuales no deseados. Estudios similares reportan prevalencias de violencia de pareja entre $20 \%$ a $85 \%$ en mujeres jóvenes y adolescentes (Eshelman \& Levendosky, 2012; García, 2013; López-Cepero et al., 2015; Martín-Baena et al., 2016; Stöckl et al., 2014; Rodríguez-Franco et al 2016; Vara-Horna et al., 2016; Viejo, 2014). Esto sugiere que la violencia a la mujer por parte de su pareja varón se está dando en edades tempranas y que no es necesario la cohabitación para que se produzca este tipo de maltrato, lo cual es preocupante dado las consecuencias que implican este problema de salud pública.

Una de las variables relacionadas a la violencia de pareja es la dependencia emocional (Aiquipa, 2015; Castelló, 2005; 2012; Huerta et al, 2016; Momeñe, Jáuregui, \& Estévez, 2017; Moral et al., 2017; Zalapa et al 2012). Si bien la dependencia emocional es concebida como la dimensión disfuncional del rasgo de personalidad necesidad de vinculación afectiva, y como tal puede ser característica tanto de hombres y mujeres, algunos estudios reportan que la mujeres tienden a reportar mayores niveles de dependencia emocional que los varones en la relación de pareja (Alonso-Arbiol, Shaver, \& Yárnoz, 2002; Avci Çayir \& Kalkan, 2018; Del Castillo et al., 2015; Rusby, Harris, \& Tasker, 2013; Sanathara, Gardner, Prescott, \& Kendler, 2003). En el estudio se encontró que el grupo de mujeres con violencia de pareja presentó puntajes más altos de dependencia emocional respecto al grupo de comparación. Así, las participantes con dependencia emocional experimentan necesidad de acceder a la pareja; sienten angustia al no estar cerca de la pareja y ante una eventual separación; priorizan a la pareja, ante todo, asumiendo conductas de sumisión y subordinación (Santamaría et al., 2015), características que podrían explicar la tolerancia de malos tratos en la relación.

Por otro lado, se halló que el grupo de mujeres con violencia de pareja se sentía menos satisfecha con la vida respecto al grupo de comparación. Dado que la relación de pareja representa un área importante en la vida de las personas pues constituye una fuente de bienestar para sus miembros (Schimmack et al., 2002), siempre y cuando esta relación sea funcional, es posible que la satisfacción en esta área se transfiera a las demás y se perciba como una satisfacción general. Por el contrario, cuando la relación es conflictiva y perjudicial, como lo es violencia de pareja, la percepción de satisfacción con la vida decrementa. Al respecto, García et al. (2014) encontraron que formas de maltrato como humillación y violencia de género tienen un efecto negativo en el bienestar psicológico.

Asimismo, se halló relación de tipo inversa entre dependencia emocional y satisfacción con la vida en ambos grupos, aunque en el grupo sin violencia fue menos intensa respecto al grupo con violencia de pareja. Esto sugiere que patrones disfuncionales de vinculación afectiva, tales como deseos de control y dominio, miedo e intolerancia a la soledad y necesidad de afecto, se asocian con baja satisfacción con la vida. Así, una persona con dependencia emocional y que sufre violencia de pareja vivirá su relación con niveles elevados de emociones displacenteras, tales como angustia, tristeza, temor, culpa, frustración e incertidumbre. Se experimenta estas emociones pues la pareja, de quien desea tener la aprobación y afecto, muestra por el contrario actitudes indiferentes, hostiles e incluso violentas. Esta situación genera sintomatología depresiva y ansiosa en la persona (Rusby et al., 2013; Sanathara et al., 2003; Santamaría et al. 2015; Urbiola et al., 2017), lo cual puede influir en su percepción de felicidad y satisfacción con la vida. 
En este contexto, es preciso señalar que una de las influencias más importantes en la satisfacción con la vida de las personas en general, y de los estudiantes universitarios en particular, es el apoyo social (Diener \& Diener McGavran, 2008; Turner \& Brown, 2010). Algunos estudios encontraron que estudiantes universitarios que obtienen una alta puntuación en la satisfacción con la vida presentan mayor apoyo social recibido y/o percibido (Novoa \& Barra, 2015; RuvalcabaRomero et al., 2017; Trepte et al., 2015), lo cual implica que perciben que cuentan con amigos y familiares cercanos de apoyo; mientras que aquellos que no tienen amigos cercanos y familiares o perciben menor apoyo social, tienen más probabilidades de sentirse insatisfechos (Diener \& Diener McGavran, 2008). Por ello, una persona con dependencia emocional y que se encuentra en una relación de pareja violenta tendrá más probabilidades de sentirse insatisfecho porque tiende a aislarse de su grupo social de soporte, esto es familiares y amigos, pues prioriza a la pareja sobre cualquier cosa. Además, cuando se instala la violencia en una relación, poco a poco el miembro agresor ejerce un control coercitivo que va limitando los contactos sociales de la víctima (Stark, 2007), incluso sin que ellos convivan. Así, el agresor suele poner a prueba el amor que le tiene la pareja haciéndola elegir entre él o los familiares y amigos de la víctima, y dado las características de dependencia emocional que tiene la mujer, optará por distanciarse de su grupo social (Zalapa et al., 2012).

Además de las relaciones interpersonales, otro factor que influye en los juicios de satisfacción con la vida en los estudiantes universitarios es el desempeño que puedan tener en sus actividades académicas (Pavot \& Diener, 2008; Samaha \& Hawi, 2016; Schimmack et al., 2002). Cuando una persona está inmersa en una relación de pareja violenta y tiende a ser dependiente de la pareja, es posible que descuide este tipo de actividades justamente porque prioriza a la pareja y hará lo que sea por mantener la relación, pues teme que la misma termine. En estas condiciones, las participantes experimentan preocupación constante, niveles elevados de angustia, estrés, malestar consigo mismas y otras emociones desagradables que podrían influir negativamente en su rendimiento académico y en consecuencia en la satisfacción con su vida.

El tener objetivos o metas personales y avanzar hacia ellas, así como el disponer de actividades de entretenimiento y de ocio, contribuyen a que una persona se sienta satisfecha con su vida (Heckhausen \& Kay, 2018; Klug \& Maier, 2015; Liu \& Yu, 2015; Pascucci, 2015). Sin embargo, en el caso de las participantes del grupo con violencia de pareja, a diferencia del grupo sin violencia, la elevada necesidad de acceso a la pareja, deseos de exclusividad y las conductas de subordinación y sumisión, generan postergación de sus metas personales y desplazamiento de sus actividades recreativas, lo que conlleva a la insatisfacción personal.

Los resultados obtenidos pueden ser útiles para las universidades nacionales y privadas ubicadas en Lima Metropolitana, las mismas que cuentan con un gran número de alumnas mujeres que estudian la carrera de Psicología y que pueden encontrarse en inmersas en situación de violencia de pareja. Dado los hallazgos, y en el marco de la política nacional intersectorial para la prevención de la violencia hacia a la mujer, las universidades pueden implementar, en sus programas de bienestar universitario, sesiones preventivas para fomentar relaciones de pareja equilibradas, funcionales y saludables para sus integrantes, así como habilidades interpersonales y de afrontamiento que fomenten una mayor satisfacción con la vida, promoviendo así factores de protección que reduzcan la probabilidad de que su población estudiantil de mujeres inicien o mantengan relaciones de pareja violentas.

Finalmente, es importante señalar que el estudio se limitó a indagar la relación entre dependencia emocional y satisfacción con la vida en las dos muestras de estudio, con y sin violencia. Por ello, no se analizó el efecto conjunto de las variables mencionadas sobre la varianza de violencia de pareja en las participantes. Asimismo, no se compararon las diferencias en las variables de estudio según el tipo de violencia (p. e., físico, psicológico 1y sexual). A partir de los aportes resultantes del estudio, investigaciones posteriores pueden indagar los aspectos referidos. 


\section{Referencias}

Aiquipa, J. J. (2012). Diseño y validación del inventario de dependencia emocional-IDE. Revista de Investigación en Psicología, 15(1), 133-145. doi: http://doi.org/10.15381/rinvp.v15i1.3673

Aiquipa, J. J. (2015). IDE. Inventario de dependencia emocional. México: El Manual Moderno.

Aiquipa, J. J. (2015). Dependencia emocional en mujeres víctimas de violencia de pareja. Revista de Psicología, 33(2), 411-437.

Aizpurua, E., Caravaca-Sánchez, F., \& Stephenson, A. (2018). Victimization status of female and male college students in Spain: prevalence and relation to mental distress. Journal of Interpersonal Violence. doi: https://doi.org/10.1177/0886260518802848

Alarcón, R. (2000). Variables psicológicas asociadas con la felicidad. Persona, 3, 147-157. doi: http://doi.org/10.26439/persona2000.n003.810

Alonso-Arbiol, I., Shaver, P. R., \& Yárnoz, S. (2002). Insecure attachment, gender roles, and interpersonal dependency in the Basque Country. Personal Relationships, 9(4), 479-490. doi: http://doi.org/10.1111/1475-6811.00030

Arias-Gallegos, W. L., Huamani-Cahua, J. C., \& Caycho-Rodríguez, T. (2018). Satisfacción con la vida en escolares de la ciudad de Arequipa. Propósitos y Representaciones, 6(1), 351407. doi: http://dx.doi.org/10.20511/pyr2018.v6n1.206

Atienza, F. L., Pons, D. Balaguer, I. \& García-Merita, M. (2000). Propiedades Psicométricas de la Escala de Satisfacción con la Vida en Adolescentes. Psicothema, 12(2), 314-319.

Ato, M., López, J. J., \& Benavente, A. (2013). Un sistema de clasificación de los diseños de investigación en psicología. Anales de Psicología, 29(3), 1038-1059. doi: http://doi.org/10.6018/analesps.29.3.178511

Avci Çayir, G., \& Kalkan, M. (2018). The effect of interpersonal dependency tendency on interpersonal cognitive distortions on youths. Journal of Human Behavior in the Social Environment, 1-16. http://doi.org/10.1080/10911359.2018.1458681

Baker, L. R., Cobb, R. A., Mcnulty, J. K., Lambert, N. M., \& Fincham, F. D. (2016). Remaining in a situationally aggressive relationship: The role of relationship self-efficacy. Personal Relationships, 23(3), 591-604. doi: http://doi.org/10.1111/pere.12145

Banyard, V. L., Demers, J. M., Cohn, E. S., Edwards, K. M., Moynihan, M. M., Walsh, W. A., \& Ward, S. K. (2017). Academic correlates of unwanted sexual contact, intercourse, stalking, and intimate partner violence: An understudied but important consequence for college students. Journal of Interpersonal Violence. doi: http://doi.org/10.1177/0886260517715022

Bernardo, A. B. I., \& Estrellado, A. F. (2017). Subjective well-being of filipino women who experienced intimate partner violence: a person-centered analysis. International Journal for the Advancement of Counselling, 39(4), 360-376. doi: http://doi.org/10.1007/s10447017-9303-1

Callahan, M. R., Tolman, R. M., \& Saunders, D. G. (2003). Adolescent dating violence victimization and psychological well-being. Journal of Adolescent Research, 18(6), 664681. doi: http://doi.org/10.1177/0743558403254784

Capaldi, D. M., Knoble, N. B., Shortt, J. W., \& Kim, H. K. (2012). A systematic review of risk factors for intimate partner violence. Partner Abuse, 3(2), 231-280. doi: http://dx.doi.org/10.1891/1946-6560.3.2.231

Castelló, J. (2005). Dependencia emocional. Características y tratamiento. España: Alianza Editorial.

Castelló, J. (2012). La superación de la dependencia emocional. Málaga: Ediciones Corona Borealis.

Caycho-Rodríguez, T., Ventura-León, J., García, C. H., Barboza-Palomino, M., Arias, W. L., Dominguez-Vergara, J.,... \& Samaniego, A. (2018). Evidencia psicométrica de la Escala de Satisfacción con la Vida en adultos mayores peruanos. Revista Ciencias de la Salud, 16(3), 488-506. doi: http://dx.doi.org/10.12804/revistas.urosario.edu.co/revsalud/a.7267. 
Cecilia, N., Martínez, M, \& Pilar M. (2013). Propiedades psicométricas de la Escala de Satisfacción con la Vida de Diener. Revista Argentina de Clínica Psicológica, 22(2), 161168.

Cohen. J. (1988). Statistical power analysis for the behavioral sciences (2nd ed.). New York: Lawrence Erlbaum Associates.

Cortés-Ayala, L., Flores, M., Bringas, C., Rodríguez-Franco, L., López-Cepero, J. y RodríguezDíaz, F. J. (2015). Relación de maltrato en el noviazgo de jóvenes mexicanos. Análisis diferencial por sexo y nivel de estudios. Terapia Psicológica, 33, 5-12. doi: http://dx.doi.org/10.4067/S0718-48082015000100001

Del Castillo, A., Hernández, M. E., Romero, A., \& Iglesias, S. (2015). Violencia en el noviazgo y su relación con la dependencia emocional pasiva en estudiantes universitarios. PSICUMEX, 5(1), 4-18.

Diener, E., Emmons, R.A., Larsen, R.J., \& Griffin, S. (1985). The Satisfaction with Life Scale. Journal of Personality Assessment, 49(1), 71-75. doi: http://doi.org/10.1207/s15327752jpa4901_13

Diener, M. L., \& Diener McGavran, M. B. (2008). What makes people happy? A developmenatl approach to the literature on family relationships and well-being. In M. Eid \& R. J. Larsen (Eds.), The science of subjective well-being (pp. 347-375). New York: Guilford.

Eshelman, L., \& Levendosky, A. A. (2012). Dating violence: Mental health consequences based on type of abuse. Violence and Victims, 27(2), 215-228. doi: http://dx.doi.org/10.1891/0886-6708.27.2.215

Estévez, A., Chávez-Vera, M. D., Momeñe, J., Olave, L., Vázquez, D., \& Iruarrizaga, I. (2018). The role of emotional dependence in the relationship between attachment and impulsive behavior. Anales de Psicología, 34(3), $438 . \quad$ doi: http://dx.doi.org/10.6018/analesps.34.3.313681

Ferguson, C. J. (2009). An effect size primer: a guide for clinicians and researchers. Professional Psychology: Research and Practice, 40(5), 532-538. doi: http://dx.doi.org/10.1037/a0015808

García, V., Fernández, A., Rodríguez, F. J., López, M.L., Mosteiro, M. P. y Lana, A. (2013). Violencia de género en estudiantes de enfermería durante sus relaciones de noviazgo. Atención Primaria, 45(6), 290-296. doi: https://doi.org/10.1016/j.aprim.2012.11.013

García, F. E., Wlodarczyk, A., Reyes, A., San Cristóbal, C., \& Solar, C. (2014). Violencia en la pareja, apoyo social y bienestar psicológico en adultos jóvenes. Ajayu, 12(2), 246-265.

George, D., \& Mallery, P. (2016). IBM SPSS Statistics 21 step by step: a simple guide and reference (14th ed.). New York: Routledge.

Gonzalez-Bueso, V., Santamaría, J.J., Merino, L., Montero, E., Fernández, D., \& Ribas, J. (2018). Dependencia emocional en mujeres: una revisión de la literatura empírica. Psicosomática y Psiquiatría, 5, 40-53.

Grissom, R. J. (1994). Probability of the superior outcome of one treatment over another. Journal of Applied Psychology, 79(2), 314-316. doi: http://dx.doi.org/10.1037/0021-9010.79.2.314

Grissom, R. J., \& Kim, J. J. (2012). Effect sizes for research: Univariate and multivariate applications (2nd ed.). New York, NY: Routledge.

Heckhausen, J. \& Kay, J. S. (2018). Motives, goals, and well- being throughout the lifespan. En J. E. Maddux (Ed.), Subjective well- being and life satisfaction. New York: Routledge. doi: http://doi.org/10.4324/9781351231879-20

Heise L. L. (2011). What works to prevent partner violence? An evidence overview. London: STRIVE Research Consortium.

Heise, L. \& García-Moreno, C. (2003). La violencia en la pareja. En Krug E. G. et al. (Eds.), Informe mundial sobre la violencia y la salud (pp. 95-131). Washington, D.C.: Organización Panamericana de la Salud.

Huerta, R., Ramírez, N., Ramos, J., Murillo, L., Falcón, C., Misare, M., \& Sánchez, J. (2016). Esquemas cognitivos disfuncionales y dependencia emocional en mujeres con y sin violencia en la relación de pareja de la ciudad de Lima. Revista de Investigación en Psicología, 19(2), 145-162. doi: http://doi.org/10.15381/rinvp.v19i2.12895 
Instituto Nacional de Estadística e Informática (2017). Encuesta demográfica y de salud familiarENDES 2017. Lima: Autor.

Klug, H. J. P, \& Maier, G. W. (2015). Linking goal progress and subjective well-being: A metaanalysis. Journal of Happiness Studies, 16(1), 37-65. doi: http://dx.doi.org/10.1007/s10902-013-9493-0

Liu, H., \& Yu, B. (2015). Serious leisure, leisure satisfaction and subjective well-being of Chinese university students. Social Indicators Research, 122(1), 159-174. doi: https://doi.org/10.1007/s11205-014-0687-6

López-Cepero, J., Lana, A., Rodríguez-Franco, L., Paíno, S. G., \& Rodríguez-Díaz, F. J. (2015). Percepción y etiquetado de la experiencia violenta en las relaciones de noviazgo juvenil. Gaceta Sanitaria, 29(1), 21-26. doi: http://dx.doi.org/10.1016/j.gaceta.2014.07.006

López-Ortega, M., Torres-Castro, S., \& Rosas-Carrasco, O. (2016). Psychometric properties of the Satisfaction with Life Scale (SWLS): secondary analysis of the Mexican Health and Aging Study. Health and Quality of Life Outcomes, 14(1), 170. doi: http://doi.org/10.1186/s12955-016-0573-9

Martín-Baena, D., Talavera, M., \& Montero-Piñar, I. (2016). Interpersonal violence and health in female university students in Spain. Journal of Nursing Scholarship, 48(6), 561-568. doi: http://doi.org/10.1111/jnu.12239

Mohamed, L. \& Herrera, L., (2014). Bienestar psicológico, autoconcepto y relación de pareja en estudiantes universitarios. International Journal of Developmental and Educational Psychology. Revista INFAD de Psicología., 6(1), 475-484. doi: http://doi.org/10.17060/ijodaep.2014.n1.v6.767

Momeñe, J., Jáuregui, P., \& Estévez, A. (2017). El papel predictor del abuso psicológico y la regulación emocional en la dependencia emocional. Psicología Conductual, 25(1), 65.

Montero, I., Escriba, V., Ruiz-Perez, I., Vives-Cases, C., Martín-Baena, D., Talavera, M., \& Plazaola, J. (2011). Interpersonal violence and women's psychological well-being. Journal of Women's Health, 20(2), 295-301. doi: http://doi.org/10.1089/jwh.2010.2136

Moral, M. V., García, A., Cuetos. G., \& Sirvent. C. (2017). Violencia en el noviazgo, dependencia emocional y autoestima en adolescentes y jóvenes españoles. Revista Iberoamericana de Psicología y Salud, 8(2), 96-107. doi: http://doi.org/10.23923/j.rips.2017.08.009

Newcombe, R. G. (2006). Confidence intervals for an effect size measure based on the MannWhitney statistic. Part 1: general issues and tail-area-based methods. Statistics in Medicine, 25(4), 543-557. doi: https://doi.org/10.1002/sim.2323

Niño, D., C., \& Abaunza, N. (2015). Relación entre dependencia emocional y afrontamiento en estudiantes universitarios. Revista Electrónica Psyconex, 7(10), 1-27.

Novoa, C., \& Barra, E. (2015). Influencia del apoyo social percibido y los factores de personalidad en la satisfacción vital de estudiantes universitarios. Terapia psicológica, 33(3), 239-245. doi: http://dx.doi.org/10.4067/S0718-48082015000300007

Padrós, F., Gutiérrez, C. Y. \& Medina, M. A. (2015). Propiedades psicométricas de la Escala de Satisfacción con la Vida (SWLS) de Diener en población de Michoacán (México). Avances en Psicología Latinoamericana, 33(2), 223-232. doi: http://dx.doi.org/10.12804/apl33.02.2015.04

Pascucci, M. (2015). Los jóvenes universitarios y el ocio. European Scientific Journal, ESJ, $11(10), 116-127$.

Pavot, W., \& Diener, E. (2008). The Satisfaction with Life Scale and the emerging construct of life satisfaction, The Journal of Positive Psychology, 3(2), 137-152. doi: http://dx.doi.org/10.1080/17439760701756946

Poutiainen, M., \& Holma, J. (2013). Subjectively evaluated effects of domestic violence on wellbeing in clinical populations. ISRN Nursing, 2013. doi: http://dx.doi.org/10.1155/2013/347235

Rodríguez-Franco, L., López-Cepero, J., López-Núñez, M. I., Paíno-Quesada, S. G., AntuñaBellerín, M. Á., Bringas-Molleda, C., \& Rodríguez-Díaz, F. J. (2016). Evolution of victimization, tolerance and detection of intimate partner violence among young Spanish women/Evolución de victimización, tolerancia y detección de la violencia de pareja entre 
mujeres jóvenes españolas. Revista de Psicología Social, 31(1), 137-156. doi: http://dx.doi.org/10.1080/02134748.2015.1101316

Rodríguez-Franco, L., López-Cepero Borrego, J., Rodríguez-Diaz, F. J., Bringas-Molleda, C., Antuña-Bellerín, M. A., \& Estrada-Pineda, C. (2010). Validación del Cuestionario Violencia entre Novios (CUVINO) en jóvenes hispanohablantes: análisis de resultados en España, México y Argentina. Anuario de Psicología Clínica y de la Salud, 6, 43-50.

Ruvalcaba-Romero, N. A., Fernández-Berrocal, P., Salazar-Estrada, J. G., \& Gallegos-Guajardo, J. (2017). Positive emotions, self-esteem, interpersonal relationships and social support as mediators between emotional intelligence and life satisfaction. Journal of Behavior, Health \& Social Issues, 9, 1-6. doi: http://doi.org/10.1016/j.jbhsi.2017.08.001

Rusby, J. S., Harris, J. M., \& Tasker, F. (2013). Female interpersonal dependency: Genetic and environmental components and its relationship to depression as a function of age. Aging \& mental health, 17(8), 1044-1051. doi: http://doi.org/10.1080/13607863.2013.807421

Samaha, M., \& Hawi, N. S. (2016). Relationships among smartphone addiction, stress, academic performance, and satisfaction with life. Computers in Human Behavior, 57, 321-325. doi: http://doi.org/10.1016/j.chb.2015.12.045

Sanathara, V. A., Gardner, C. O., Prescott, C. A., \& Kendler, K. S. (2003). Interpersonal dependence and major depression: Aetiological inter-relationship and gender differences. Psychological Medicine, 33(5), 927-931. doi: http://doi.org/10.1017/S0033291703007542

Santamaría, J. J., Merino, L., Montero, E., Cano, M., Fernández, T., Cubero, P., ... \& GonzálezBueso, V. (2015). Perfil psicopatológico de pacientes con Dependencia Emocional. Cuadernos de Medicina Psicosomática y Psiquiatria de Enlace, (116), 36-46.

Schimmack, U., Diener, E., \& Oishi, S. (2002). Life-satisfaction is a momentary judgment and a stable personality characteristic: The use of chronically accesible and stable sources. Journal of Personality, 70, 345-384. doi: http://doi.org/10.1111/1467-6494.05008

Stark, E. (2007). Coercive control: How men entrap women in personal life. Oxford: Oxford University Press.

Stöckl, H., March, L., Pallitto, C., \& Garcia-Moreno, C. (2014). Intimate partner violence among adolescents and young women: prevalence and associated factors in nine countries: a crosssectional study. BMC public health, 14(1), 751. doi: http://doi.org/10.1186/1471-2458-14751

Trepte, S., Dienlin, T., \& Reinecke, L. (2015). Influence of social support received in online and offline contexts on satisfaction with social support and satisfaction with life: A longitudinal study. Media Psychology, 18(1), 74-105. doi: http://doi.org/10.1080/15213269.2013.838904

Turner, R. J., \& Brown, R. L. (2010). Social support and mental health. In T. Scheid \& T. Brown (Eds.), A handbook for the study of mental health: Social contexts, theories, and systems (pp. 200-212). New York, NY: Cambridge University Press. doi: http://doi.org/10.1017/CBO9780511984945.014

United Nations (2015). The World's Women 2015: Trends and Statistics. New York: Autor.

Urbiola, I., \& Estévez, A. (2015). Dependencia emocional y esquemas desadaptativos tempranos en el noviazgo de adolescentes y jóvenes. Behavioral Psychology, 23(3), 571-587.

Urbiola, I., Estévez, A., Iruarrizaga, I., \& Jauregui, P. (2017). Dependencia emocional en jóvenes: relación con la sintomatología ansiosa y depresiva, autoestima y diferencias de género. Ansiedad y Estrés, 23(1), 6-11. doi: http://dx.doi.org/10.1016/j.anyes.2016.11.003

Vara-Horna, A., López-Odar, D., Alemán, L., Asencios, Z., Bailón, Y., Bayona, B..., Ventura, Ruth. (2016). La violencia contra las mujeres en las universidades peruanas. Prevalencia e impacto en la productividad académica en las facultades de ciencias empresariales e ingeniería. Lima: GIZ \& USMP.

Vera-Villarroel, P., Urzúa, M. A., Pavez, P., Celis-Atenas, K. \& Silva, J. (2012). Evaluation of subjective well-being: Analysis of the satisfaction with life scale in Chilean population. Universitas Psychologica, 11(3), 719-727. doi: http://doi.org/10.11144/Javeriana.upsy113.eswa

Viejo, C. (2014). Physical dating violence: towards a comprehensible view of the phenomenon/Violencia física en las relaciones sentimentales adolescentes: hacia la 
comprensión del fenómeno. Infancia y Aprendizaje, 37(4), 785-815. doi: https://doi.org/10.1080/02103702.2014.977110

Zalapa, E. E., García, Y. E. \& Figueroa, G. E. (2012). Resiliencia en mujeres morelianas separadas por violencia conyugal. Revista de Psicología, 14(2), 164-177.

\section{Financiamiento}

El presente estudio fue auspiciado y financiado por el Consejo Superior de Investigación de la Universidad Inca Garcilaso de la Vega. 